International Journal of Engineering \& Technology, $7(3.8)(2018) 151-154$
International Journal of Engineering \& Technology
Website $: \frac{w w w . s c i e n c e p u b c o . c o m / i n d e x . p h p / I J E T}{2}$
Research paper

\title{
Introduction to Bigdata and Relation with IoT
}

\author{
Anjali Deore ${ }^{1 *}$ \\ Departement of Computer Engineering, Sandip Foundation Nashik,SPPU, Maharashtra, India \\ *E-mail: anjalideore13@gmail.com
}

\begin{abstract}
Big Data consist of large scale data which is complicated and diverse, so that new and different types of integration of techniques and technologies are required to uncover various hidden values from such big datasets. Big Data surrounding is used to set up and examine the diverse sorts of information. Big Data be data that is so massive in volume, so various in range or moving with excessive speed is referred to as Big Data. Acquiring and analysing Big Data be a challenging job because it consists of large dispersed file systems which must be bendy, fault tolerant and scalable. Diverse technologies used by big data application toward hold the huge quantity of data are Hadoop, Map Reduce, and so on. In this paper, firstly the description of big dataset is provided. In next section the different technologies are described which are used for managing Big Data. After that, Big Data method application and in last section we discuss the relation of Big Data and IoT as well as IoT for Big Data analytics.
\end{abstract}

Keywords: Big data, Hadoop , MapReduce, Internet of Things, Analytics..

\section{Introduction}

In these days environment, data is generating from diverse sources. This information is of various varieties. Capturing, retrieving, extracting, analyzing, manipulating and storing of these data is bit essential. This amount of big facts is taken into consideration as Big Data. Big data is described as facts which isn't always only very big, however additionally excessive in speed and variety, which makes them hard to deal with the use of traditional gear and techniques. These data is generated from exceptional social media like Twitter, Facebook and so forth., from special transactions accomplished in corporation's databases, from supply chain situations which materials tons of statistics, as an instance given quantity of scanners and so on. There are five demanding situations of huge facts control are: volume, range, pace, price, veracity.

A. Volume: Information is always expanding day by day of the whole kinds still MB, PB, YB, ZB, KB, TB of data. The information results into colossal records. How to store the large volume of data?, is a basic issue now a days. This essential issue is settled through bringing down capacity esteem. Data size is increasing every day by 50 instances by methods for 2020.

B. Variety: Information assets are heterogeneous. The archives comes in various code and of different forms, it could be based or unorganized including log reports, sound, recordings, printed content. The sorts are endless, and the information provided to the system while not having been measured or qualified in any way.

C. Velocity: The information comes at intemperate speed. Some of the time 60 seconds essentially too past due so enormous it is time touchy data. A few ventures information speed is essential task.
D. Value: It is extreme fundamental $\mathrm{v}$ in large information. Esteem is fundamental buzz for large size data as it is critical for offices, IT framework contraption to store large size of virtues in database.

E. Veracity: The blast in the assortment of qualities basic of a major informational collection. When we overseeing high amount, speed and sort of information, the all of data are not going precise, there can be messy information. Enormous data and investigation innovation works of art with those sorts of information [12].

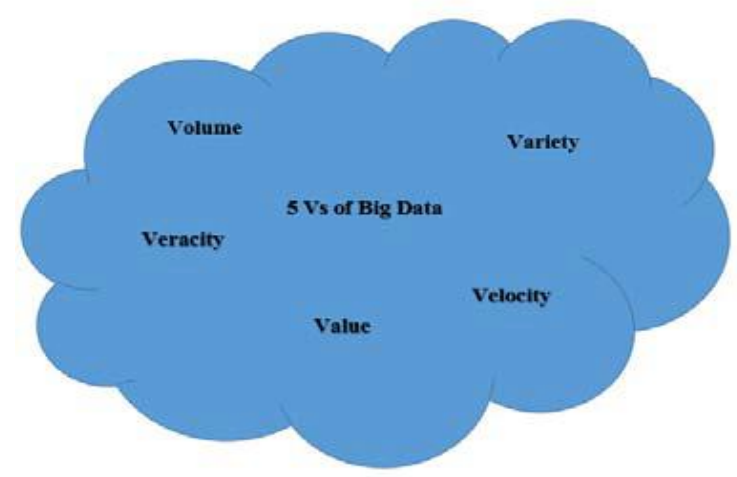

Fig1. Challenges of Big Data

In this paper, a evaluation on Big Data is represented which incorporates meaning of big data, various challenges of big data. Section 2 offers the literature survey which is based totally on Big Data. Section 3 introduces one of a kind technologies used for dealing with Big Data. Section 4 consists of application of Big Data in distinct fields. In Last, section 5 IoT for Big Data Analytics. 


\section{Literature Survey}

AThe author [1] stated definition of big data and different parameters of big data. The author has also explained different technology with their advantages and disadvantages and lastly explains the applications of big data.

The author [2] stated different challenges of big data. The author has also explained different technology architecture with their advantages and disadvantages.

Yuri Demchenko [3] stated that Hadoop Map Reduce is an open supply, large scale software program framework which is devoted to distributed, scalable, data-in depth computing. This framework first break up huge data into smaller chunks so as to be parallel and switch arrangement. It maps each piece to an intermediate price the use of Map characteristic after which reduces intermediate values to an answer using Reduce function. So Map Reduce is a great manner to remedy the ones issues in which big dataset may be broken into smaller portions parallel in a distributed environment.

Amogh Pramod Kulkarni et.al [4] expounded the significance of some of the advances that can be utilized for managing Big Data like Hadoop, HDFS and Map Reduce. The creator said roughly various schedulers which might be used in Hadoop and around the extraordinary specialized parts of Hadoop moreover. The creator also accentuations on the significance of YARN which over forces the impediments of Map Reduce.

M. R. Berthold et.al [5] has audited one of a kind advances to hold the enormous information and its engineering. In this paper, moreover specified the difficulties and engineering of Big Data. Additionally talked about stand-out benefits and negative marks of that innovation of Big Data the utilization of ongoing NoSQL databases, Hadoop HDFS appropriated information stockpiling and Map Reduce conveyed records handling over a bunch of item servers.

Sagiroglu et.al [6] expressed Big Data meaning and more grounded the definition by strategy for giving the $5 \mathrm{~V}$ Big Data living arrangements and furthermore advised diverse measurements for Big Data examination and scientific categorization, exceptionally unmistakable and assessing Big Data advancements in big business, e-Science, online networking, business, human services.

Ms. Vibhavari Chavan et.al [7] stated that information is growing unexpectedly every day. Now those information isn't always best restrained inside gigabyte, instead it's miles more than this measure. The data generated isn't always handiest too huge in length it's far heterogeneous additionally. Big Data evaluation tools like Map Reduce over Hadoop and HDFS, guarantees to assist businesses higher apprehend their clients and the marketplace, which hopefully results in competitive advantages and to higher enterprise decisions.

Research ordered, including research configuration, explore strategy (as calculations, Pseudocode or other), how to test and information securing [1]-[3]. The portrayal of the course of research ought to be bolstered references, so the clarification can be acknowledged deductively [2], [4].

\section{Technologies and Methods}

Big Data is a fresh out of the box new idea for overseeing gigantic information thusly the structural depiction of this innovation could be new. There are the excellent advances which utilize relatively indistinguishable approach that is to circulate the actualities among various nearby merchants and decrease the weight of the rule server all together that movement might be kept away from. Various papers, books and articles that depict Big Data as new era so we can rather consideration our endeavors ideal here on starting a couple of straightforward measures and the base age establishment to help relate Big Data to the more extensive IM zone.

\subsection{Hadoop}

Hadoop is a system that may run programs on structures with a great many hubs and terabytes. It disseminates the report a portion of the hubs and lets in to gadget proceed with work if there should be an occurrence of a hub disappointment. This technique decreases the danger of unfortunate framework disappointment. Amid which application is separated into minor parts. Apache Hadoop incorporates the Hadoop part, Hadoop disseminated document framework (HDFS),. Hadoop Distributed File System incorporates with Data Node, the Name Node, Secondary Name Node. Hadoop is usually utilized for appropriated cluster record building; it is legitimate to enhance the list capacity in close real time. Hadoop gives added substances to storage room and in addition investigation for huge scale handling. Presently a day's Hadoop utilized by several organizations. The advantage of Hadoop is Distributed capacity and Computational abilities, amazingly versatile, optimized for high throughput, huge piece sizes, tolerant of programming project and equipment failure.

\section{A. Hadoop Component:}

- HBase: It keeps running over the HDFS layer. It ready to give the information and yield to the Map Reduce in well expound structure..

- Oozie: It It utilize the database to gather the data of Work stream which is an accumulation of strategies. It deals with the Hadoop occupations positively.

- Sqoop: Sqoop is an organize line edge asks for that gives stage which is used to varying over information from Hadoop to social databases or the other way around.

- Avro: It is a structure that gives data serially and organization of data trade. It is fundamentally utilized as a part of Apache Hadoop.

- Chukwa: This framework is used for information social affair and investigation to process and crash the tremendous measure of logs. It is the higher level of the HDFS and Map Reduce.

- Pig: It is an abnormal state information preparing framework where the information records are broke down that happens in abnormal state dialect.

- Zookeeper: It is single point control based administrations that give dispersed organization and furnishes assemble benefits alongside upkeep of the setup data and records.

- Hive: Hive is the best layer of Hadoop that assistance in giving conclusion, and examination for particular questions Hadoop is:

- Reliable: it handles failure of hardware and software, fault tolerant occurs with software;

- Scalable: considered for huge size of processors, memory, and local storage

- Distributed: gives hugely parallel programming model, Map Reduce, handles duplication

Hadoop is of use when difficult information processing is required, Machine learning and important convention coding would be essential.

\subsection{MapReduce}

MapReduce strategy keeps huge datasets on product hardware. MapReduce is a form to prepare enormous scale data in group. The Map Reduce programming rendition is fundamentally in view of capacities which can be Map () capacity and Reduce () work. 
Map work executes as ace hub takes the info, separate into littler sub modules and disseminate into slave hubs. A slave hub extra partitions the subordinate modules afresh that demonstrate the route to the various levelled tree plan. The slave hub methods the base inconvenience and passes the outcome again to the ace Node The Map Reduce machine mastermind together all halfway combines based at the middle of the road keys and allude them to Reduce () work for delivering the last yield. Reduce work functions as the ace hub gathers the impacts from the greater part of the sub issues and joins them all things considered to structure the yield.

Map Reduce Components:

- Name Node:handle HDFS metadata, doesn't compact with files at once.

- Data Node: stores blocks of HDFS, default duplication phase for each block.

-Job Tracker: schedules, allocates and monitors job execution on slaves.

- Task Tracker: executes MapReduce functions.

\subsection{Hive}

Hive is a scattered specialist stage, a decentralized framework for structure applications by means of systems administration nearby framework assets. Apache Hive information warehousing part and part of cloud-based Hadoop biological method which offers an examination dialect called Hive QL that proselytes SQL queries into Map Reduce employments mechanically. Utilizations of apache hive are SQL, prophet, IBM DB2. Design is isolated into Map-Reduce-arranged usage, Meta data for information stockpiling, and an implementation part to get a question from customer. The benefit of hive is additional ensured and executions are high calibre. The downside of hive is only for impromptu inquiries and execution is less when contrasted with pig.

\subsection{No-SQL}

No-SQL database is a procedure to data administration and realities design that is useful for to great degree colossal arrangements of appropriated information. These databases are in standard a piece of the continuous occasion which may be identified in technique allowing age subsequent diagnostic abilities comprehensive of relative inquiry programs. These are most ideal made because of the lithe idea of the No-SQL show wherein the dimensionality of a question is created from the data in extension and space in inclination to being settled with the guide of the engineer ahead of time. The upside of No-SQL is open supply, level adaptability, Easy to utilize, store confounded insights composes, Very quick to add new records and for basic activities/questions. The drawback of No-SQL is Immaturity, No ordering keep up, No ACID, composite unwavering quality models, Absence of institutionalization.

\subsection{HPCC}

It is an open source stage use to register and that offers the bearer for adapting to of huge data work process. HPCC information variant is characterized by means of the individual quit steady with the necessities. HPCC machine is proposed after which correspondingly intended to control the most mind boggling and records inside and out expository related issues. HPCC machine is a solitary stage having a solitary design and a solitary programming dialect utilized for the information reproduction. HPCC machine transformed into intended to look into the critical amount of information for the reason of understanding confounded issue of huge information. HPCC machine is fundamentally in view of office control dialect which has the revelatory and on-procedural nature training dialect the rule added substances of HPCC will be: HPCC Data processing unit: Use analogous ETL motor for the most part. HPCC Data Delivery: It is inconceivably in view of organized inquiry motor utilized.

\section{Applications}

In the present time, due to large quantity of heterogeneous data, there is exceptional call for of Big Data. Big data is widely used in lots of applications. Some applications are as follows-

1. Public Sector: Huge amount of data is producing from various resources with high speed. For managing that information, Big Data is used. Big Data presents better insights of unstructured and dependent data. It affords smart decision by right danger evaluation. This is the purpose why all of the groups are inclined in the direction of Big Data. Big Data provides a huge variety of centers to the authorities sectors which include deceit reputation, fitness interconnected exploration, the strength research, ecological fortification and economic promotion research.

2.

Health care: The large information has wide use inside the field of healthcare and medicine. With the improve of technology cost, the fee of health care is too increasing. Big data is contribution great helping hand on this field. It allows the physicians to preserve the track of all patient's history which can be accessed by means of the handiest the patient or his precise medical doctor. All the data associated with patients are stored securely in database for all time. There are massive wide variety of medical gadgets which are big data orientated. Today data is used to such a quantity that health practitioner recommends the medicines without even travelling the patient.

3.

Learning: Big data has exquisite impact inside the educational field. Now in recent times, almost each route of studying is gift on-line. There are many more examples of the usage of massive data inside the education enterprise. There is an utility named as the Bubble Score which lets in teachers to convey more than one-desire valuations via cellular gadgets and notch up paper exams during the cameras of the cell phones. Along with this device, there are many more techniques which can be carried out the usage of Big Data in training subject.

4.

Industrial and Natural Resources: Big data provide solutions for natural sources too. The excessive volume in addition to the rate of big data is challenged by using the excessive call for of the herbal sources on this earth. The unused data avoids power competence, superior eminence of merchandise, advanced profits obstacles and dependability. In the herbal wealth industry, big data empowers for analytical modelling to preserve judgment advent this is used to contain and devour huge quantities of information from graphical data, geographical facts, chronological and manuscript data.

5.

Banking Zones and Fraud Detection: In the banking sectors, Big Data is hugely used within the fraud detection. Big Data unearths out all of the mischief responsibilities achieved inside the banking sectors. It detects the misuse of debit cards, misuse of credit playing cards, assignment credit risk remedy, archival of inspection tracks, patron facts alteration, business readability, IT motion analytics, public analytics for business and IT method fulfillment analytics [8] [9] [10]. In banking zone, at present they are the use of natural speech processors and community analytics to grasp unlawful commercial enterprise interest inside the economic marketplaces. In corporations huge facts enables loads in knowing CRM techniques of the competition and the shopping patterns of clients with a view to placed on them of their agencies so one can enhance the sales.

\section{The IoT and Big Data: Making the Relation}

Big Data is about volume, velocity, variety, value. But at the end of the day, it's all about data. IoT is about data, device and connectivity. In future more objects and devices are connected to the 
Internet and exchange as well as gather the information for data analysis. So,data (big or small) is always important and center part of the connecred devices in IoT World.

The intersection of IoT and Big Data is creating a tremendous business opportunity and also help to leans about patterens and trends of our lifestyle, heathcare, agriculture etc. In agriculture field, big data and IoT both contribute huge. The moisture level of field monitor by the field connects systems and this system transmits recorded moisture level to farmers by using a wireless connection. With the help of this information farmers are able to find out when the optimum moisture levels for th crop. Big Data analytics are a key revenue opportunity in IoT Sector. Some analysts indicate that by $202040 \%$ of data will come from sensors.

\section{IoT for Big Data Analytics}

The idea of IoT is turning into more pertinent to the sensible global due to the development of mobile devices, embedded and ubiquitous communication technology, cloud computing, and statistics analytics. An IoT device generates non-stop streams of records and the researchers can broaden tools to extract meaningful data from these data the use of gadget learning strategies. Therefore, it is critical to develop infrastructure to research the IoT records. Knowledge acquisition from IoT information is the biggest mission that massive data professional are going through. Understanding these streams of records generated from IoT devices and analysing them to get meaningful statistics is a challenging issue and it ends in massive statistics analytics. Machine gaining knowledge of algorithms and computational intelligence strategies is the simplest solution to deal with large facts from IoT potential.

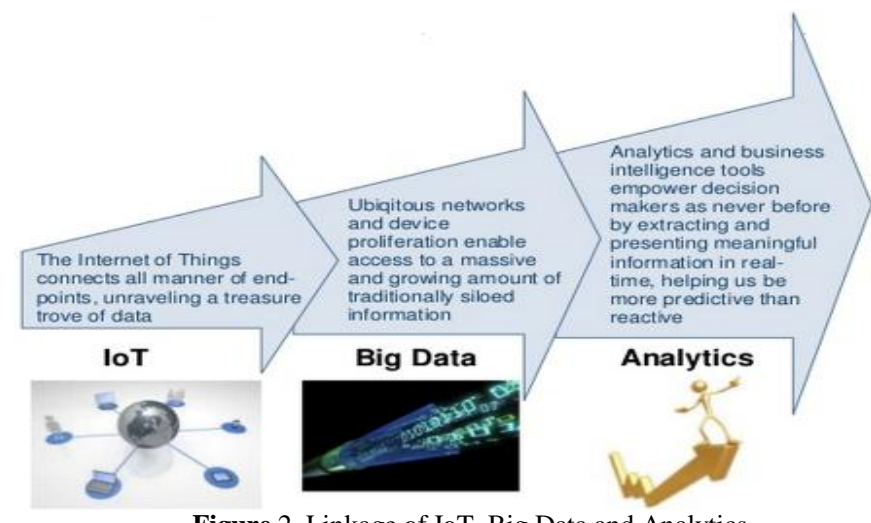

Figure 2. Linkage of IoT, Big Data and Analytics

Knowledge exploration structures have originated from theories of human data processing like frames, rules, tagging, and linguistics networks. In general, it consists of 4 segments like information acquisition, cognitive content, information dissemination, and information application. In knowledge acquisition phase, expertise is located with the aid of using various conventional and computational intelligence strategies. The discovered know-how is saved in knowledge bases and professional structures are usually designed based totally on the determined understanding. Knowledge dissemination is critical for obtaining meaningful records from the information base Knowledge extraction is a process that searches files, information within documents in addition to know-how bases. The final section is to apply discovered know-how in diverse packages. It is the final word goal of information discovery [11].

\section{Conclusion}

In this paper idea of Big Data and numerous technologies has been surveyed that are used take care of the large information. The essential goal of this paper changed into toward create a review of diverse Big Data structure, its dealing with techniques which cope with a huge amount of records from distinct resources and improves general performance of systems and its applications which shows its significance and makes use of within the present IT international

\section{References}

[1] Tasleem Nizam and Syed Imtiyaz Hassan, "Big Data: A Survey Paper on Big Data Innovation and its Technology," in International Journal of Advanced Research in Computer Science, Vol.8, No. 5, pp. 2173-2177, 2017.

[2] C. Lakshmi and V. V. Nagendra Kumar "Survey Paper on Big Data," in International Journal of Advanced Research in Computer Science and Software Engineering, vol.6, No. 8, pp. 368-381, 2016.

[3] Yuri Demchenko, "The Big Data Architecture Framework (BDAF)", Outcome of the Brainstorming Session at the University of Amsterdam 17 July 2013.

[4] Amogh Pramod Kulkarni, Mahesh Khandewal, "Survey on Hadoop and Introduction to YARN", International Journal of Emerging Technology and Advanced Engineering Website: www.ijetae.com (ISSN 2250-2459, ISO 9001:2008 Certified Journal, Volume 4, Issue 5, May 2014).

[5] M. R. Berthold, N. Cebron, F. Dill, T. R. Gabriel, T. Kötter, T. Meinl, et al., "KNIME: The Konstanz Information Miner", in Data Analysis, Machine Learning and Applications (Studies in Classification, Data Analysis, and Knowledge Organization), Springer Berlin Heidelberg, pp. 319-326, 2008.

[6] Sagiroglu, S.Sinanc, D.,'Big Data: A Review”,2013, 20-24.

[7] Ms. Vibhavari Chavan, Prof. Rajesh and N. Phursule, "Survey Paper On Big Data", International Journal of Computer Science and Information Technologies, Vol. 5 (6), 2014

[8] Samiddha Mukherjee and Ravi Shaw, "Big Data - Concepts, Applications, Challenges and Future Scope", International Journal of Advanced Research in Computer and Communication Engineering, 2016.

[9] Hua Fang, Zhaoyang Zhang, ChanpaulJin Wang, Mahmoud Deshmand, Chonggang Wang, and HonggangWang, "A Survey of Big Data Research", IEEE Network, 2015.

[10] KuchipudiSravanthi and TatireddySubba Reddy, "Applications of Big Data in Various Fields", International Journal of Computer Science and Information Technology, 2015.

[11] D. P. Acharjya, Kauser Ahmed P, "A Survey on Big Data Analytics: Challenges, OpenResearch Issues and Tools", International Journal of Advanced Computer Science and Applications, Vol. 7, No. 2, 2016

[12] Anjali Deore, Bhayashree More, Kaveri Sonawane, Jyoti Kharat, "Introduction to Hadoop Architecture and Installation on Ubantu", International Journal of Research in Engineering and Technology, Vol. 6, issue 9, 2017. 